\title{
Crystal engineering and the design of higher cocrystals
}

Niyaz A. Mir ${ }^{1}$, Ritesh Dubey², Gautam R. Desiraju²

${ }^{1}$ Department Of Chemistry, Women's College, Aligarh Muslim University, Aligarh, India, ${ }^{2}$ Solid State and Structural Chemistry Unit, Solid State and Structural Chemistry Unit, Bangalore, India

E-mail: mirniyaz@gmail.com

Crystallization is an ancient method of purification based on a phenomenon that excludes rather than includes. Thus, the synthesis of multi-component crystals is a challenging task and their isolation is often unpredictable. Obtaining a multicomponent crystal means that one is able to calibrate and assess intermolecular interactions rather carefully. The scope of crystal engineering is now well explored towards the designing of crystalline binary cocrystals. However, increasing the number of components in a cocrystal is a crystal engineering equivalent of synthetic complexity. Cocrystallization of a mixture of four compounds could, in principle, give not only binaries but also a number of ternaries. Ultimately, it would appear that the level and degree of control to design a quaternary cocrystal is formidable. This presentation will highlight the fact that through crystal engineering principles, we can design the four- and even higher-component molecular crystals. A concise synthetic strategy has been applied to increase the number of components in a crystal from one to two, to three, to four and eventually to five. Ten stoichiometric quaternary cocrystals and also one quintinary cocrystal having partial evidence of solid solution are designed. The strategy is based on the exploitation of the different environments of any particular component in a cocrystal.

[1] Mir. N. A. et al. (2015) CrystEngComm 17, 7866-7869.

[2] Mir. N. A. et al. (2016) IUCr] 3, 96-101.

[3] Dubey. R. et al. (2016) IUCr] 3, 102-107.

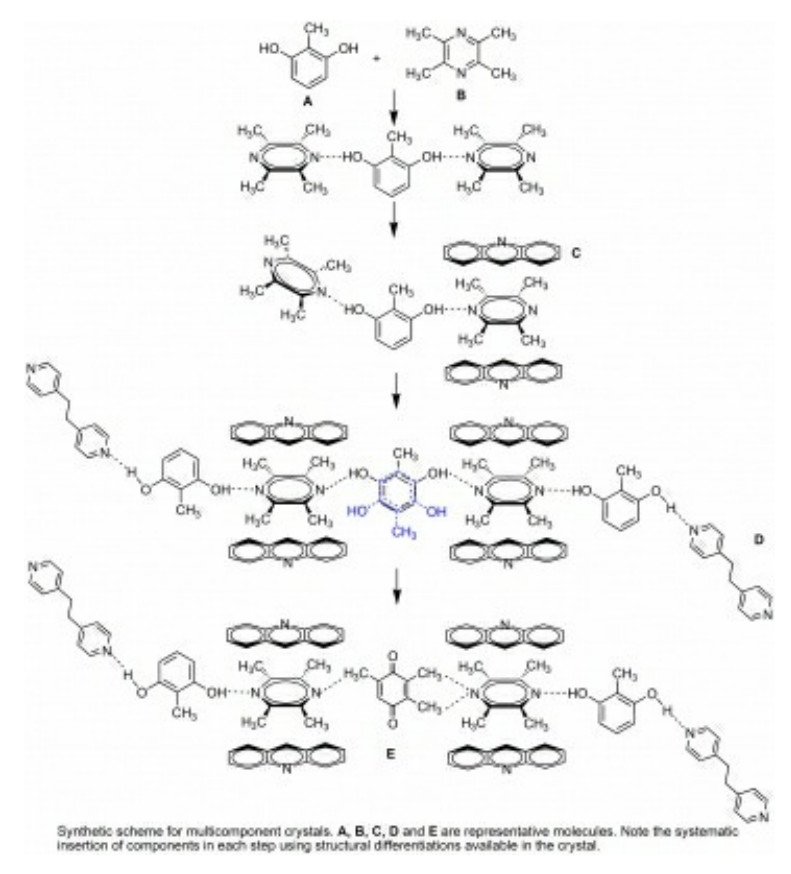

Keywords: Crystal engineering, intermolecular interactions, cocrystals 